Horizons philosophiques

\title{
En quel sens faut-il entendre la formule de Gilles Deleuze voulant que la philosophie soit une création de concepts?
}

\section{Charles Bolduc}

Volume 17, numéro 1, automne 2006

Existentialisme et philosophie continentale

URI : https://id.erudit.org/iderudit/802966ar

DOI : https://doi.org/10.7202/802966ar

Aller au sommaire du numéro

Éditeur(s)

Collège Édouard-Montpetit

ISSN

1181-9227 (imprimé)

1920-2954 (numérique)

Découvrir la revue

\section{Citer cet article}

Bolduc, C. (2006). En quel sens faut-il entendre la formule de Gilles Deleuze voulant que la philosophie soit une création de concepts? Horizons philosophiques, 17(1), 47-68. https://doi.org/10.7202/802966ar d'utilisation que vous pouvez consulter en ligne. 


\section{En quel sens faut-il entendre la formule de Gilles Deleuze voulant que la philosophie soit une création de concepts?}

De toutes les prises de position qui ont ponctué sa vie et son oeuvre, Gilles Deleuze est largement connu aujourd'hui pour sa définition de la philosophie comme «art de former, d'inventer, de fabriquer des concepts'1. II est vrai qu'à l'aune de l'histoire de la pensée, la philosophie semble bien avoir toujours été ce constant travail de renouvellement des concepts. Cependant, il ne faut pas oublier le caractère polémique de cette assertion qui se veut en rupture avec une tradition dont les tenants ont répugné à concevoir que des idées par eux seuls créées puissent avoir sens et valeur eu égard aux exigences qu'il prêtent à leur pratique. En procédant à une relecture de certaines problématiques de l'histoire de la philosophie à la lumière de la position défendue par Gilles Deleuze, l'objectif de cet article est d'éclairer tous les enjeux inhérents à cette définition. Il va débuter par une mise en contexte critique de celle-ci, puis une seconde partie va mesurer sa portée ontologique et, enfin, il va se terminer par une confrontation de l'empirisme classique avec l'empirisme transcendantal qu'implique cette définition.

\section{Renversement critique nécessaire à une redéfinition de la philosophie comme création de concepts}

Afin de comprendre le sens proprement novateur de la définition deleuzienne de la philosophie, il convient tout d'abord de se demander quelle doit être l'،Image de la pensée" pour qu'il soit possible de disqualifier la valeur de la création de concepts dans l'exercice philosophique. En effet, par son parti pris, Deleuze nous force à nous interroger sur la nature d'une philosophie qui ne serait pas une création de concepts, c'est-à-dire qu'il nous invite à réfléchir sur les présupposés implicites qui discréditent de manière a priori la création comme pratique proprement philosophique. Lorsqu'il affirme que la philosophie est inséparable d'une création de concepts, Deleuze s'oppose ainsi explicitement à l'idée contraire selon laquelle un concept n'aurait pas besoin d'être créé puisqu'il ne serait qu'une simple reprise réflexive, une sorte de décalque de ce qui est, de ce qui est déjà là et qui n'attend que le moment où la pensée va daigner 
se pencher sur lui pour se révéler, tel qu'il est véritablement, sous la forme d'un concept. Ce qui est par conséquent en premier lieu critiqué et dénoncé, c'est cette fonction représentative du concept, cette "Image morale de la pensée» qui présuppose l'affinité et la complémentarité d'un "donné» premier avec une pensée destinée à le recueillir dans le concept, de telle sorte qu'il n'y aurait rien de préphilosophique qui ne serait, en bout de ligne, philosophiquement pensable.

Cette remise en question pourrait certes être menée de plusieurs fronts, mais suivant cette idée qu'il dit avoir puisée chez Nietzsche, Deleuze fait de l'étude généalogique le point de vue proprement critique : c'est en retraçant l'impensé, le lieu d'enracinement de la conception interrogée, que sa mise en perspective et son éventuel renversement vont être rendus possibles². La démarche généalogique se distingue ainsi du criticisme kantien qui cherche à fonder en droit, c'est-à-dire de manière a priori, ce qui est en question. II ne faut donc pas confondre le principe critique revendiqué par Deleuze avec le criticisme kantien qui en vient malgré tout, sous la dénomination d'«idées régulatrices", à légitimer le «Moi», le «Monde» et «Dieu»3. C'est ce que Deleuze entend lorsqu'il affirme par exemple que : «Le premier principe de la philosophie est que les Universaux n'expliquent rien, ils doivent être expliqués» 4 . Ce même principe critique sera appliqué au discrédit général dévolu à la création en philosophie : mettre en lumière les présupposés qui induisent ce jugement, et ce, pour voir ce qu'eux-mêmes présupposent en dernière instance comme point de vue fondateur.

Dans le troisième chapitre de Différence et répétition, Deleuze définit l' «Image morale de la pensée» précédemment évoquée par huit postulats. Ceux-ci constituent les présupposés subjectifs implicites qui supportent la définition d'une philosophie qui se veut sans commencement, c'est-à-dire sans création d'aucune sorte :

10 postulat du principe, ou de la Cogita natura universalis [...]; 20 postulat de l'idéal, ou du sens commun [...]; 3ำ postulat du modèle, ou de la recognition [...]; $4^{\circ}$ postulat de l'élément, ou de la représentation [...]; 50 postulat du négatif, ou de l'erreur [...]; $6^{\circ}$ postulat de la fonction logique, ou de la proposition [...]; $7^{0}$ postulat de la modalité, ou des solutions [...]; $8^{2}$ postulat de la fin ou du résultat, postulat du savoir

Parmi ceux-ci, c'est évidemment le quatrième postulat, celui de 
la représentation, qui est le plus décisif en ce qui a trait à la place assignée à la création de concepts en philosophie. Celui-ci a quatre caractéristiques :

L'élément de la représentation comme "raison» a quatre aspects principaux : l'identité dans la forme du concept indéterminé, l'analogie dans le rapport entre concepts déterminables ultimes, l'opposition dans le rapport des déterminations à lintérieur du concept, la ressemblance dans l'objet déterminé du concept lui-mêmé.

Alors que le premier postulat assure l'adéquation de la pensée à ce qui se présente à elle, ce quatrième postulat fait du concept l'instance qui réduit, intériorise et subordonne la différence potentielle qui peut exister entre la pensée et son dehors. Tout d'abord, puisque le concept représente "un» objet, il doit avoir une identité, c'est-à-dire une unité a priori inaliénable, même si aucun contenu sensible particulier n'est encore subsumé sous lui. Deuxièmement, puisqu'il y a tout de même une diversitě de concepts déterminés, les concepts déterminables "ultimes" (les catégories d'Aristote par exemple7) doivent être analogues, et ce, afin de préserver, au sein de la diversité, l'homogénéité et l'unité de la pensée dans la représentation qu'elle se fait d' «un» monde. Troisièmement, étant donné qu'une représentation est une reprise réflexive de ce qui est, alors la distinction entre le sensible et l'intelligible se voit résorbée à l'intérieur du concept en une opposition de déterminations. Enfin, pour que l'on puisse départager le vrai du faux, le concept doit d'une certaine façon pouvoir être dit correspondre à l'objet qu'il représente, ce qui est rendu possible quand on fait de l'objet lui-même un concept élevé en référent type à partir duquel la similitude est perçue, mesurée et jugée.

On voit bien à partir des deux derniers points que, dans la représentation, la différence n'est plus qu'une différence intérieure au concept et la création, un exercice de différenciation conceptuelle. Conséquemment, sous de tels présupposés, aucune véritable création de concepts n'est possible, puisque ne pourra être pensé que ce qui est potentiellement contenu dans un concept déjà formé et posé comme fondement. La fonction représentative va donc ultimement de pair avec cette subordination du fondé (sensible) au fondement (intelligible, conceptuel).

Selon Deleuze, Platon fut le premier à jeter les bases de cette 
réduction du sensible à sa condition de légitimité. Dans le Sophiste, Platon ne se contente pas de distinguer l'Idée (intelligible) de sa copie (sensible), car cela ne lui est pas suffisant pour départager les différents prétendants à la vérité. II lui faut, dans un deuxième temps, distinguer la copie du simulacre, la bonne de la mauvaise copie ${ }^{8}$. Ainsi, c'est parce que la première participe de l'ldée et que la seconde ne s'y réfère pas que cette dernière peut être disqualifiée comme n'ayant aucun fondement de légitimité possible. La véritable différence, la différence déterminable, assignable, ne passe donc plus entre deux ordres hétérogènes (le sensible et l'intelligible), mais entre le fondement (l'Idée) et ce qui est fondé par lui (la copie) ${ }^{9}$. C'est finalement parce que la différence est rapportée à l'ldée que le produit de la création, le simulacre, peut être discrédité comme ne produisant rien d'intelligible ou même de simplement compréhensible.

Cependant, bien qu'inlassablement critiquée et rejetée, une différence sensible, non conceptuelle, subsiste pourtant toujours chez Platon et celle-ci revient constamment hanter le philosophe. Selon l'interprétation qu'en tire Deleuze, cette différence n'est en fait pleinement neutralisée et résorbée dans le concept qu'à partir du moment où la pensée se fait explicitement représentation et la représentation, infinie : «La représentation infinie comprend précisément une infinité de représentations, soit qu'elle assure la convergence de tous les points de vue sur un même objet ou un même monde, soit qu'elle fasse de tous les moments les propriétés d'un même Moi»10.

D'une part, il importe peu que ce centre de convergence conceptuel soit le "Monde» ou le «Moi», puisque de toute façon, toute représentation est nécessairement une reconnaissance subjective de l'adéquation des déterminations avec le concept. En d'autres termes, que ces déterminations soient des points de vue sur le «Monde» ou les propriétés d'un «Moi», une représentation n'est telle que pour un sujet qui la pense dans un concept. La représentation infinie est ainsi inséparable d'un idéalisme subjectif : ne peut être dit exister que ce qui est pensé dans une représentation conceptuelle. L'argument semble irréfutable : comment pourrait-on en effet affirmer ou même nier l'existence de quelque chose dont on n'a aucune pensée déterminée ${ }^{11}$ ? Ce qui revient à dire que soit le sensible est une idée, un être intelligible, soit il est un mot dénué de toute signification. C'est donc précisément parce que, dans la représentation infinie, l'existence sensible se réduit à la pensée de l'existence que Deleuze qualifie de «subjectifs» les présupposés de cette «Image de la pensée». 
D'autre part, dans la représentation infinie, c'est le concept luimême qui se fait le fondement du monde. Que celui-ci soit le "Monde" ou le "Moi», qu'il soit un terme originaire d'où dérive la différence ou encore l'élément qui assure la réconciliation synthétique des contraires, dans tous les cas, la différence se voit relativisée et intériorisée dans le concept ${ }^{12}$. Il y a ici une antériorité logique du concept qui fonde en droit la représentation et réduit la différence au "Même» et au «Semblable»13. C'est donc parce qu'une définition de la philosophie comme création de concepts ébranlerait le fondement même de cet édifice qu'il y a une telle répugnance à lui accorder une quelconque valeur. On comprend mieux du coup pourquoi c'est une "Image morale de la pensée»: puisque toute pensée ne peut que dériver d'un concept déjà pensé, alors n'aura de valeur que ce qui est déjà reconnu, c'est-à-dire ce qui a déjà valeur en cours.

Nous arrivons ici au cœur de l'enquête généalogique : l'impensé, le point d'ancrage de la représentation, c'est la négation de l'autonomie de la différence et sa subordination au concept posé comme fondement du monde. Celui-ci fait alors de la différence soit une opposition intérieure au concept, soit un contenu indéterminé, indifférencié, qui ne prend réellement corps que dans ce concept. Renverser ce fondement, c'est renverser cette alternative qui fait du fondé une dépendance du fondement, de telle sorte que ce soit dorénavant la différence, c'est-à-dire le point de vue différentiel qui soit le domaine propre de la pensée.

Ce point de départ, Deleuze le qualifie à juste titre de critique, car il ne s'agit pas de substituer un fondement à un autre, ni de faire de la création un concept, mais de poser la création de concepts comme mouvement nécessaire à tout avènement de la pensée ${ }^{14}$. La critique de cette "Image» est ainsi le renversement critique de tout fondement :

Dès lors apparaissent mieux les conditions d'une philosophie qui serait sans présupposés d'aucune sorte : au lieu de s'appuyer sur l'Image morale de la pensée, elle prendrait son point de départ dans une critique radicale de l'Image et des "postulats" qu'elle implique. Elle trouverait sa différence ou son vrai commencement non pas dans une entente avec l'Image préphilosophique, mais dans une lutte vigoureuse contre I'Image, dénoncée comme non-philosophie ${ }^{15}$.

C'est précisément en questionnant cette correspondance, cette 
entente présupposée entre le "donné» et le concept que Deleuze en est venu, à la suite de Nietzsche $^{16}$, à intimement lier la pratique philosophique et la création de concepts alors définie comme le surgissement de la différence, comme l'éternel retour de la différence. Puisque l'autonomie et la valeur intrinsèque de la création sont d'emblée disqualifiées par une certaine "Image de la pensée» qui réduit et subordonne constamment la pratique philosophique à la fonction représentative d'un «donné» premier, une reconsidération de la portée philosophique de la création passe nécessairement par une revalorisation de la différence, du surgissement toujours renouvelé de concepts au sein même de la philosophie. Deleuze ne remet donc pas en question les concepts philosophiques en tant que tels (l'Idée platonicienne, le Cogito cartésien, etc.), mais il opère seulement une réévaluation critique qui les projette sous une nouvelle lumière.

\section{Portée ontologique de la définition deleuzienne de la philosophie}

Ainsi, une fois mis au jour le point de vue fondateur qui s'oppose à une définition de la philosophie comme création de concepts, Deleuze entend le renverser en faisant de la différence même l'objet propre du concept philosophique. II va sans dire que l'«Image de la pensée» change dès lors radicalement de sèns, car ce qui est donné à penser, ce n'est plus l'être, mais le devenir'17. La nature du concept s'en voit d'autant transformée : il ne se veut plus une représentation de la chose, mais la chose comme point de vue, "l'être problématique" de la chose envisagée sous le signe de sa différence constitutive :

Le préfixe RE- dans le mot représentation signifie cette forme conceptuelle qui se subordonne les différences. Ce n'est donc pas en multipliant les représentations et les points de vue, qu'on atteint à l'immédiat défini comme «sub-représentatif». Au contraire, c'est déjà chaque représentation qui doit être déformée, déviée, arrachée à son centre. II faut que chaque point de vue soit lui-même la chose, ou que la chose appartienne au point de vue. II faut donc que la chose ne soit rien d'identique, mais soit écartelée dans une différence où s'évanouit l'identité de l'objet vu comme du sujet voyant ${ }^{18}$.

L' «être» est alors cette différence qui, en se posant, pose problème. Du coup, le non-être n'est plus une négation de l'être, mais «l'être du problématique»: «Ce (non)-être est l'Élément différentiel où l'affirmation, comme affirmation multiple, trouve le principe de sa 
genèse»19. Si une chose est étudiée du point de vue de sa genèse, de son affirmation comme phénomène, alors elle ne peut plus être considérée comme un "donné», mais elle doit être envisagée comme un mixte d'hétérogènes, comme une rapport «d'ordres de différence» qui conditionne son actualisation:

Tout phénomène renvoie à une inégalité qui le conditionne. Toute diversité, tout changement renvoient à une différence qui en est la raison suffisante. Tout ce qui passe et qui apparaît est corrélatif d'ordres de différence : différence de niveau, de température, de pression, de tension, de potentiel, différence d'intensitế20.

II est important de souligner que, pour Deleuze, l'intensité n'est jamais une quantité absolue, une quantité toujours constante et invariablement déterminée, mais seulement une différence entre deux points qui sont eux-mêmes les produits d'autres différences entre deux points, eux-mêmes les résultats respectifs de deux autres rapports différentiels, etc. (à l'infini), de sorte qu'une intensité exprimée ne peut jamais être claire (c'est-à-dire enveloppant et expliquant une différence) sans être aussi en même temps confuse (enveloppée, impliquée par d'autres différences) ${ }^{21}$. Ces points singuliers ne sont donc jamais purement et simplement «donnés», puisqu'ils sont déterminés d'après la totalité virtuelle des rapports différentiels dont ils n'en actualisent qu'un, dont ils ne peuvent en exprimer clairement qu'un à la fois. Conséquemment, la différence d'intensité n'est pas une différence entre deux moments déterminés, mais le point de jonction de l'indéterminé et du déterminé22. Autrement, si la différence pouvait se mesurer par la comparaison directe de deux déterminations, alors elle ne serait plus un mixte d'hétérogènes, mais seulement une différence conceptuelle, une opposition de déterminations à l'intérieur d'un concept déjà formé.

L'épreuve de l'éternel retour de la différence doit se comprendre ainsi : sous l'apparent retour du "Même" et du "Semblable», du $f$ ondement dans le fondé, du possible dans le réel, c'est seulement la différence qui revient. Ce qui est semblable et identique dans l'éternel retour de la différence, c'est la différence elle-même qui revient et qui se répète. En effet, dans l'éternel retour, seule la véritable différence est affirmée, car ce qui reste le même, ne changeant pas, demeure indéterminé, indifférencié dans le devenir. À l'aune de l'éternel retour, on se rend bien compte que la différence conceptuelle n'est pas une 
véritable différence, car les variations intérieures au concept ne le font pas varier lui-même. La différence conceptuelle est donc incapable d'expliquer le retour de la différence, la création d'un nouveau concept, l'engendrement du "penser" dans la pensée»23.

Au contraire, si une "chose" est un point de vue, alors l'ajout d'un seul élément change radicalement celle-ci et rend nécessaire la création d'un nouveau concept ${ }^{24}$. C'est pourquoi l'éternel retour est une épreuve et un processus de sélection : la différence n'est plus intérieure au concept, mais entre deux formes de répétition, entre celle, illusoire, qui fait advenir la distinction numérique dans le concept et celle qui crée à l'inverse la distinction formelle entre concepts 25 . C'est du coup l'éternel retour de la différence qui permet de rendre compte du caractère illusoire du "Même» et du «Semblable» quand ils sont intériorisés dans le concept :

Ce renversement n'est pas seulement spéculatif, il est éminemment pratique puisqu'il définit les conditions de légitimité de l'emploi des mots identique et semblable en les liant exclusivement aux simulacres, et dénonce comme illégitime l'usage ordinaire qui en est fait du point de vue de la représentation ${ }^{26}$.

Finalement, c'est parce que l'éternel retour de la différence est capable de rendre compte des mixtes d'hétérogènes que la différence est dite plus profonde que le supposé fondement de la représentation qui demeure foncièrement inapte, à partir de ses présupposés, d'élucider le processus de détermination d'un contenu sensible (réel) sous un concept (possible)27. En d'autres termes, c'est parce que le fondement est incapable d'expliquer comment le fondé en vient à être subsumé sous lui qu'il peut être légitimement renversé. Ainsi, les présupposés "subjectifs" de la représentation n'apparaissent plus comme des "erreurs" injustifiables ou inexplicables, mais comme des «illusions", n'étant tout compte fait qu'une sorte d'oubli masqué de la profondeur d'où ils sont nés 28 . Conséquemment, la représentation n'est plus qu'un point de vue qui se nie comme point de vue, une actualisation qui nie la virtualité qu'elle implique ${ }^{29}$.

Comparaison de l'empirisme transcendantal deleuzien avec l'empirisme classique de Locke, Berkeley et Hume : de quelle manière doit-on comprendre que la création de concepts est redevable du sensible?

À la recherche d'un fondement forcément illusoire, Deleuze substitue la création de concepts rendant compte, non de la réalité 
des phénomènes proprement dits, mais de l'être des phénomènes dans leur nature problématique. II n'est jamais question chez Deleuze de la connaissance d'un être sensible particulier, mais seulement de la chose même comme différence, comme point de vue différentiel. La question n'est plus de penser le sensible, ni de savoir comment le sensible peut être conditionné par l'intelligible. Ce qui est en jeu dans la création de concepts, c'est l' «être du sensible» :

Il est étrange qu'on ait pu fonder l'esthétique (comme science du sensible) sur ce qui peut être représenté dans le sensible. Ne vaut pas mieux, il est vrai, la démarche inverse qui soustrait de la représentation le pur sensible, et tente de le déterminer comme ce qui reste une fois la représentation ôtée (par exemple un flux contradictoire, une rhapsodie de sensations). En vérité l'empirisme devient transcendantal, et l'esthétique, une discipline apodictique, quand nous appréhendons directement dans le sensible ce qui ne peut être que senti, l'être même du sensible : la différence, la différence de potentiel, la différence d'intensité comme raison du divers qualitatif. C'est dans la différence que le phénomène fulgure, s'explique comme signe, et que le mouvement se produit comme "effet». Le monde intense des différences, où les qualités trouvent leur raison et le sensible, son être, est précisément l'objet d'un empirisme supérieur ${ }^{30}$.

Ainsi, en ce qu'il ouvre la voie à une définition de la philosophie comme création de concepts, l'empirisme supérieur, «transcendantal», est le renversement critique de la philosophie comme volonté de représentation. II est vrai que cette expression d' «empirisme transcendantal» peut paraître de prime abord paradoxale et c'est pourquoi nous allons conclure ce parcours par une comparaison de celui-ci avec l'empirisme classique de Locke, Berkeley et Hume. Cette confrontation avec une tradition reconnue pour avoir destitué bon nombre de concepts métaphysiques de leur piédestal souverain nous permettra de préciser concrètement en quel sens il faut entendre qu'il y a création de concepts en philosophie.

Tout d'abord, les rapports de Deleuze à l'empirisme classique sont complexes et ambigus. D'une part, il s'en réclame en rappelant constamment que "la philosophie manque d'empirisme»31 ou encore que l'empirisme est un "grand créateur de concepts»32. Mais d'autre part, il ne cesse de relever les insuffisances des recherches purement 
"empiriques»33. Sous cette dernière dénomination, Deleuze se réfère à une conception éculée de l'empirisme qui fait de toutes nos idées la représentation ou le décalque d'un supposé "donné» premier. Mais qu'en est-il vraiment de ce «donné» dans l'empirisme classique?

Dès les premières pages de l'Essai philosophique concernant l'entendement humain de Locke, le "donné» s'avère un donné problématique. En effet, Locke nous prévient que même si son objet d'étude est l'entendement humain, son but n'est pas de révéler les secrets de la constitution de l'esprit34, ni une fois connus les rouages et le fonctionnement de celui-c,i de plonger dans les méandres de l'être 35 . C'est donc dire que Locke abandonne le questionnement ontologique sur la nature première (ou dernière) des choses et que la sensation, le «donné» sensible auquel réfèrent nos idées n'est, ontologiquement parlant, absolument rien et que, par conséquent, les concepts que nous en formons n'ont aucune légitimité a priori.

Dans l'empirisme classique, le retour à l'origine première des idées n'a pas pour fonction de confirmer ou d'infirmer une idée en tant qu'être de la chose, c'est-à-dire en tant que reprise conceptuelle adéquate de la chose perçue par les sens. Le recours à l'expérience est seulement un principe qui permet d'évaluer les idées entre elles et de distinguer celles qui sont scientifiquement recevables de celles qui ne veulent tout simplement rien dire. En d'autres mots, si une idée ne peut supporter cette épreuve généalogique, alors elle ne doit pas être considérée comme une véritable «idée» : «Toute idée que l'esprit n'a jamais perçue, n'a jamais été dans l'esprit»36. Cependant, malgré ce principe discriminatoire qui permet d'y voir un peu plus clair dans notre esprit, il est toujours impossible de savoir si une idée représente ou non un «objet» en tant que tel.

II est vrai que Locke n'est pas toujours clair sur cette question de la représentation. D'un côté, il se fait le pourfendeur acharné des idées innées cartésiennes, mais de l'autre, il s'en fait l'héritier en maintenant la distinction entre qualités secondes et qualités premières, et en faisant de ces dernières de véritables reproductions intellectuelles des choses telles qu'elles sont en elles-mêmes indépendamment de nous :

les idées des qualités premières des corps ressemblent à ces qualités, et les exemplaires de ces idées existent réellement dans les corps, mais les idées, produites en nous par les qualités secondes, ne leur ressemblent en aucune manière, et il n'y a rien dans les corps mêmes qui ait de la conformité avec ces idées ${ }^{37}$. 
Locke ajoute aussitôt que, même si ces qualités premières échappent parfois à nos sens, elles n'en sont pas moins réelles : "ll y a réellement dans le feu ou dans la neige des parties d'une certaine grosseur, figure, nombre et mouvement, que nos sens les perçoivent ou non : c'est pourquoi ces qualités peuvent être appelées réelles, parce qu'elles existent réellement dans ces corps»38. Ce qui est étrange, c'est que le principe de l'expérience semble justement contredire ce statut dévolu aux qualités premières. En effet, s'il n'est pas possible de retracer dans l'expérience l'origine d'une idée, si une idée se dérobe à cette enquête, alors rien ne nous pousse à l'admettre et tout à la rejeter. C'était d'ailleurs sur cet argument que s'appuyait Locke pour remettre en cause le caractère inné des idées cartésiennes ${ }^{39}$. Cette contradiction n'échappera pas à Berkeley qui en fera la critique dans de nombreux passages de ses Dialogues.

Berkeley interprète cette reconnaissance de l'existence de qualités premières comme une concession faite aux partisans de la fonction représentative de l'idée. Cette conception n'est cependant pas rationnellement défendable. L'argument de Berkeley est le suivant : puisque notre expérience du monde se résume à une série de perceptions, c'est-à-dire à une succession d'idées qui défilent dans notre esprit, et que la chose elle-même ne serait justement pas une idée, il nous est impossible de savoir si ces idées que nous avons ressemblent aux supposées qualités premières qui constitueraient l'essence stable de la chose ${ }^{40}$. D'où le fameux "être c'est être perçu» 41 : c'est parce qu'elle ne pourra jamais être perçue en tant que telle que l'idée d'une qualité première n'a aucun sens.

La démarche de Hume s'inscrit pour une bonne part dans la veine de ces deux prédécesseurs. Dès l'introduction de son Traité de la nature humaine, Hume nous dit, comme Locke, qu'il compte s'en tenir à l'expérience et qu'il ne visera pas à dégager les ressorts ultimes de l'esprit humain 42 . Et à l'instar de Berkeley, il va faire une critique acérée des qualités premières en démontrant que, puisque nous n'avons toujours droit qu'à des perceptions, l'idée d'un objet existant indépendamment de nous ne peut prétendre à aucune légitimité ${ }^{43}$. De plus, même à supposer qu'un tel objet existerait, nous ne pourrions jamais avoir l'expérience de la relation que celui-ci entretient avec les perceptions qu'il induit en nous. Ce qui veut dire que nous ne pourrions jamais savoir si ces perceptions ressemblent à l'objet, puisqu'une telle comparaison entre l'objet et les perceptions serait elle-même une perception ${ }^{44}$. Enfin, dernier argument, puisque toutes 
nos perceptions sont éphémères, l'expérience ne nous offre pas l'occasion d'avoir des idées de qualités premières et d'objets toujours identiques à eux-mêmes à travers le temps. Ces idées sont donc de pures fictions de l'imagination 45 .

Toutefois, un problème persiste : si toute chose n'est qu'une idée, s'il est impossible de savoir si une idée ressemble à l'objet qui l'a suscitée, s'il est même impossible, à la limite, de parler de l'identité de cet "objet», comment peut-on alors en venir à départager le rêve de la réalité, les productions de l'imagination de la connaissance véritable?

Dans l'empirisme classique, ce qui distingue le savoir légitime de celui qui ne l'est pas, c'est le sentiment d'adhésion que cette idée provoque en nous, c'est la force de conviction qu'elle infère dans notre esprit. En d'autres termes, la connaissance est une croyance et non une certitude fondée sur la fonction représentative de l'idée. Chez Locke, ce qui est dit réellement exister, c'est ce qui s'impose à nous :

Lors, dis-je, que ces idées particulières [dérivées de l'expérience sensorielle d'un corps] se présentent à l'esprit, l'entendement n'a pas la puissance de les refuser ou de les altérer lorsqu'elles ont fait leur impression, de les effacer ou d'en produire de nouvelles en lui-même, non plus qu'un miroir ne peut point refuser, altérer ou effacer les images que les objets produisent sur la glace devant laquelle ils sont placés ${ }^{46}$.

À la lecture de ce passage, on pourrait encore croire que ce qui est connu, c'est un «donné», un «objet» dont on a l'idée. Il faut cependant nous rappeler que dans l'empirisme classique, on a moins affaire à des connaissances d'objets qu'à des relations d'idées : "La connaissance consistant [...] dans la perception de la convenance ou de la disconvenance de nos idées»47. L'extériorité des relations par rapport à leurs termes est ainsi, après celui de l'expérience, le deuxième principe de l'empirisme classique ${ }^{48}$. Nos rêves par exemple n'ont aucune valeur au niveau de la connaissance, parce que les relations d'idées qu'ils présentent brisent le cours ordinaire de l'expérience et provoquent de ce fait un certain malaise en nous. Ce dernier est alors la cause de notre incrédulité 49 . L'idée n'est donc jamais la représentation d'un objet "réel» et ce qui provoque l'adhésion, le sentiment de "réalité", c'est seulement la conformité des idées et des liens qu'elles entretiennent entre elles avec la cohérence et la stabilité des expériences passées.

Avec Berkeley, le problème est posé dans toute son acuité. On aurait pu penser de prime abord qu'en démontrant qu'il n'existe pas 
d'objets en tant que tels, Berkeley avait résolu la question du réalisme de l'épistémologie lockienne. Or, il n'en est rien. En effet, même s'il n'existe que des idées, il y en a parfois qui sont contradictoires entre elles : comment alors distinguer celles qui sont légitimes de celles qui ne le sont pas? La réponse de Berkeley n'est pas tellement différente de celle proposée par Locke : comme les productions de l'imagination dépendent de notre volonté et qu'elles sont ainsi susceptibles de multiples transformations arbitraires, elles ne s'imposent pas implacablement à nous comme c'est le cas de celles que nous offre l'expérience courante ${ }^{50}$. C'est donc finalement toujours ces dernières qui gagnent notre confiance et qui sont qualifiées de «réelles» : «il faut l'avouer, ces créatures de la fantaisie ne sont pas du tout aussi distinctes, aussi fortes, aussi vives et permanentes que celles qui sont perçues par mes sens, lesquelles sont appelées choses réelles»51.

La position de Hume sur ces questions n'est décidément pas différente de ces deux prédécesseurs lorsqu'il fait de la vivacité le critère pour départager ce qui est scientifiquement recevable de ce qui ne l'est pas : «ll apparaît ainsi que la croyance ou l'assentiment qui accompagne toujours la mémoire et les sens n'est rien d'autre que la vivacité des perceptions qu'ils présentent, et que cela seul les distingue de l'imagination „52. Comme Berkeley, Hume considère que la vivacité accompagne l'idée qui est conforme à l'expérience courante. Une idée sera donc d'autant plus forte et crédible qu'elle aura cette consistance et cette régularité que l'on prête à ce dont on a quotidiennement l'expérience : "Une idée qui reçoit l'assentiment, nous l'éprouvons comme différente d'une idée fictive que la fantaisie nous présente. Et cette différence, je m'efforce de l'expliquer par ce que j'appelle une force, une vivacité, une solidité, une fermeté, ou une stabilité supérieures» 53 .

Un point distingue cependant le scepticisme de Hume de la position plus dogmatique de Berkeley. Pour ce dernier, nous ne connaissons les choses que par les idées que nous avons et tout ce qui n'en est pas ou n'en perçoit pas doit alors être rejeté comme n'ayant aucun sens. Cet idéalisme va de pair avec la promotion d'une substance spirituelle comme fondement du monde. Du fait que je ne puis pas toujours disposer des idées à ma guise et que certaines d'entre elles s'imposent à moi, malgré moi, Berkeley en conclut qu'il doit y avoir un autre principe qui en soit la cause :

il est évident que les choses que je perçois sont mes propres idées, et qu'aucune idée ne peut exister si ce n'est dans un 
esprit. Et il n'est pas moins clair que ces idées ou choses que je perçois, que ce soient elles-mêmes ou leurs archétypes, existent indépendamment de mon esprit, puisque je sais que je n'en suis pas moi-même l'auteur, et qu'il est hors de mon pouvoir de déterminer à ma guise de quelles idées particulières je serai affecté en ouvrant les yeux ou en prêtant l'oreille; elles doivent donc exister dans quelque autre esprit, dont c'est la volonté qu'elles me soient montrées ${ }^{54}$.

Étant donné que, par définition, une idée est un être passif (c'està-dire perçu par un esprit), nous ne pouvons avoir une idée de ce principe actif. Cependant, puisque nous savons que nous sommes nous-mêmes un tel esprit actif (quoique fini), nous pouvons par analogie penser ce principe actif suprême et absolu de toutes les idées dont nous pouvons avoir l'expérience. Mais puisque nous le pensons de cette manière-là, indirectement, Dieu n'est pas une idée, mais une notion 55 . En affirmant cela, Berkeley rompt avec le principe de l'expérience et avec le principe de l'extériorité des relations par rapport à leurs termes. C'est pourquoi il peut ensuite conclure que les lois que l'on découvre dans la Nature sont les lois mêmes de Dieu56.

Hume est très critique quant à la possibilité de pouvoir connaître une telle substance spirituelle. Tout d'abord, dans la section $D e$ l'identité personnelle, Hume démontre, conformément au principe de l'expérience, que toute idée de "soi» est une fiction : comme nous n'avons pas d'expérience constante de nous-mêmes, alors nous ne pouvons pas en conclure qu'il existe une telle chose comme l'identité personnelle ${ }^{57}$. Sans cette dernière, nous ne pouvons penser analogiquement la notion de Dieu. Deuxièmement, après avoir démontré que la relation qui unit une cause à son effet n'est jamais elle-même l'objet d'une impression (principe de l'extériorité de la relation par rapport à ses termes), Hume en tire la conclusion logique qu'il est impossible d'avoir l'expérience de Dieu comme cause première du monde 58 . Par conséquent, il n'y a pas lieu de prêter au «moi" et à "Dieu» un caractère substantiel, même spirituel, ni de faire d'eux la cause de nos idées.

C'est donc en critiquant la substance spirituelle que Hume se déprend de l'idéalisme de Berkeley et de ses prétentions au fondement et à la totalisation de l'expérience. Ainsi, il fait de la croyance l'un des principaux concepts de l'empirisme : puisqu'il n'y a plus aucune certitude, puisque même l'existence de Dieu n'est pas 
assurée, alors toute connaissance, quelle qu'elle soit, est probable et relève d'une croyance plus ou moins fondée ${ }^{59}$. Hume va même jusqu'à affirmer qu'il est possible qu'une idée fictive soit plus vive qu'une idée tirée d'une impression de sensation 60 ou encore jusqu'à soutenir que l'on peut raisonnablement croire à un «fait» dont on n'a pas eu sensiblement l'expérience ${ }^{61}$. Locke avait déjà analysé une telle inversion du principe de l'expérience dans son chapitre Des degrés d'assentiment où il montre que ce qui était au départ douteux pour les témoins directs en vient parfois progressivement à acquérir une légitimité par le seul fait que tous y ont donné par la suite leur assentiment62.

Ces deux derniers exemples nous montrent bien que ce que Locke et Hume prêtent au cours régulier de l'expérience n'est plus limité à l'expérience personnelle d'un individu, mais embrasse l'histoire de l'humanité et de ses productions. C'est une autre raison qui nous fait rejeter l'idée que, dans l'empirisme, ce qui est légitime doit être une simple copie d'un donné sensible (ou spirituel dans le cas de Berkeley).

Deleuze avait bien relevé ce point et c'est pourquoi, en ce qui a trait à l'empirisme, il s'intéresse moins au "donné" qu'aux relations :

Ce n'est pas du tout la question "est-ce que l'intelligible vient du sensible?", mais une tout autre question, celle des relations. Les relations sont extérieures à leurs termes [...]. On voit bien comment en découle le pseudo-premier principe de l'empirisme, mais comme une limite négative toujours repoussée, un masque mis au début : en effet, si les relations sont extérieures et irréductibles à leurs termes, la différence ne peut pas être entre le sensible et l'intelligible, entre l'expérience et la pensée, entre les sensations et les idées, mais seulement entre deux sortes d'idées, ou deux sortes d'expériences, celle des termes et celle des relations ${ }^{63}$.

Nous voyons cependant qu'à la toute fin de cet extrait, Deleuze semble hésiter entre deux formulations : ce qui est en jeu dans l'empirisme, est-ce une différence d'idées ou d'expériences?

D'une part, on se rappelle que chez Hume il n'y a pas d'idées de relation : celle-ci est seulement sentie comme une propension, comme une force irrésistible qui nous pousse à joindre à l'idée présente celle, absente, qui lui est généralement associée ${ }^{64}$. S'il y avait 
de telles idées de relation, cela voudrait dire que les termes qui la composent ne sont pas réellement distincts et que l'on pourrait analytiquement (c'est-à-dire intellectuellement, rationnellement) les déduire de cette idée ${ }^{65}$. On pourrait même penser qu'il est possible de remonter jusqu'au terme ultime, jusqu'à l'idée à partir de laquelle on déduirait logiquement toutes les autres. La pensée serait alors totalement adéquate à l'être et la connaissance humaine serait ontologiquement (c'est-à-dire totalement) justifiée. Or, si Hume tient tant à cette extériorité des relations par rapport à leurs termes, c'est parce qu'elle s'oppose radicalement à la méthode déductive cartésienne et à la possibilité de telles affirmations sur l'«être» des choses.

D'autre part, l'objet propre de l'empirisme transcendantal, c'est l'idée d'un rapport différentiel. Mais cette idée ne dit pas l'être des choses qu'elle met en relation. Elle est plutôt l'expression des conditions nécessaires pour réaliser l'union de deux "idées" au sein d'un même problème. Sur ce point, il ne faut pas confondre l'empirisme transcendantal avec la philosophie transcendantale kantienne. Kant pense abstraitement l'union de deux objets, c'est-à-dire qu'il recherche seulement les conditions de possibilité de toute expérience et il demeure indifférent aux relations concrètes d'objets particuliers. La philosophie kantienne n'est pas une philosophie des relations, mais de la constitution d'objets dont le terme ultime serait le "Monde», c'est-à-dire la Nature ${ }^{66}$. Deleuze au contraire recherche les conditions réelles de la relation. Celles-ci définissent alors l'être problématique propre de cette dernière. Mais puisqu'elles ne mettent en jeu des "objets", elles ne sont pas une connaissance de la vérité de la relation. Ainsi, l'idée de la relation, l'être problématique de l'union préserve la différence formelle et l'extériorité de la relation par rapport à ses termes.

En faisant dériver les termes de la relation d'impressions de sensation, Hume ne pouvait admettre que les rapports qu'ils entretiennent entre eux soient eux-mêmes des idées ou des concepts, car cela l'aurait précipité dans une philosophie transcendantale de type kantienne qui va à l'encontre de toute son entreprise : il aurait ontologiquement légitimé le «Moi», le «Monde» et «Dieu». Pour Deleuze cependant, la différence entre le "donné» et la relation, c'est la différence entre l'Idée comme représentant de la réalité essentielle de la chose et l'Idée comme différence, comme instance problématique.

Ainsi, même s'ils partagent une lutte commune contre la tentation 
de l'idéalisme, une différence majeure subsiste entre la conception de la philosophie de l'empirisme classique et celle soutenue par Deleuze : pour Hume, l'empirisme est une différence d'expérience (de sensation et de réflexion), une différence sentie (même si ce senti n'a aucune prétention ontologique), alors que pour Deleuze, c'est une différence d'ldées, de concepts : ce qui l'intéresse, ce n'est pas le sensible, c'est l'être du sensible, c'est l'expression d'un événement, d'un devenir. Conséquemment, pour l'empirisme classique, le concept est une vérité, une proposition plus ou moins probable sur le monde (répartition des chances), alors que pour Deleuze, le concept est le problème lui-même, le coup de dés qui affirme «tout le hasard en une fois; et c'est là l'essence de ce qu'on appelle question»67.

\section{La création de concepts : d'une réflexion sur l'être à l'expres- sion d'événements}

Finalement, bien que Hume et Deleuze sapent tous deux les soubassements de la fonction représentative du concept, seul ce dernier soutient jusqu'au bout la définition d'une philosophie comme création de concepts, et ce, en parvenant à montrer par un renversement critique que toute pratique philosophique la disqualifiant la présuppose pourtant. Cette plongée dans les profondeurs de la pensée pourrait nous faire croire que Deleuze a seulement mis au jour un fondement plus originaire que tout fondement jusqu'alors pensé. Mais ce serait oublier qu'un tel "concept» de la création est en même temps "la pensée la plus pauvre et la plus vide», comme il le dit de l'accord de la Nature et des principes de la nature humaine chez Hume 68 . Tout comme cet accord n'est rien a priori puisqu'il résulte a posteriori d'une pratique qui ne fait que le confirmer, de même il n'y a pas de véritable création de concepts sans l'épreuve de l'éternel retour de la différence. II ne faut pas prendre cette «profondeur» trop au sérieux. Deleuze l'affirme d'ailleurs clairement dans Logique du sens : le sens est un effet de surface, un événement, une "entité non existante»69. En un mot : il est une création.

Comme Gilles Deleuze essayera de le concevoir avec Félix Guattari dans Mille plateaux, tous ces partages entre l'Un et le multiple, entre systèmes arborescent et rhizomatique, entre organisations molaire et moléculaire, entre le lisse et le strié, entre les strates et les lignes de fuite, entre territorialisation et déterritorialisation, tous ces partages donc ne doivent pas être pensés en termes essentialiste, ontologique ou hiérarchique (dépendance de l'un par rapport à l'autre), mais plutôt dans leur mouvement respectif et dans leur inter- 
action constante ${ }^{70}$. Tout état dit "stable» n'étant qu'un état passager se voulant plus durable que les autres, alors que tout état "passager» dénote de son côté un certain état de coagulation et de stabilité, tout n'étant finalement que degrés de contraction et de dilatation exprimant des événements, des devenirs qui ne doivent pas être confondus à des "êtres" s'inscrivant dans le déploiement de l'Histoire ${ }^{71}$. Mais n'est-ce pas alors la même chose que d'affirmer que ces séries d'oppositions strictes sont, pour reprendre la terminologie de Différence et répétition, des «illusions» et non des «erreurs»?

Enfin, il est vrai que cet article n'a donné lieu à aucune création de concepts et qu'il pourrait, en ce sens, être considéré comme un échec philosophique. Mais c'est précisément parce qu'il fallait tout d'abord renverser une certaine conception de la philosophie pour seulement pouvoir penser sa pratique comme une telle création de concepts. Certes, des concepts ont toujours besoin d'être créés pour répondre aux nouveaux problèmes qui surgissent constamment en philosophie et Deleuze n'a certainement pas été en reste sur ce point (nous n'avons qu'à penser aux concepts de littérature mineure, de rhizome, de plateau, etc.). Mais l'on peut aussi maintenant tout simplement jeter un nouveau regard sur l'histoire de la philosophie et découvrir que, sous l'apparence pompeuse de certains concepts qui se présentaient comme étant éternels et universels, c'est en fait une étonnante machine à produire des concepts qui se profile. Sous cette lumière, l'empirisme classique n'est plus le moment d'une réduction épistémologique de la connaissance (certitude) à la croyance (probable), mais l'événement que ponctue la création du concept même de croyance ${ }^{72}$. De même peut-on envisager que, chez Nietzsche et Deleuze, loin d'être le fondement et la raison dernière des concepts, l'éternel retour n'est qu'un concept qui permet de mettre en rapport deux termes qui semblaient depuis Platon tout à fait incompatibles : la création et l'Idée.

\author{
Charles Bolduc \\ Faculté de théologie, d'éthique \\ et de philosophie \\ Université de Sherbrooke
}




\section{Bibliographie}

ARISTOTE, Métaphysique (traduction de Jules Tricot), Paris, Vrin, 1991, 2 vol.

BERGSON, Henri, La pensée et le mouvant, Paris, PUF, 1938.

BERKELEY, George, Principes de la connaissance humaine (traduction de Dominique Berlioz), Paris, GF-Flammarion, 1991.

-, Trois dialogues entre Hylas et Philonous (traduction de Geneviève Brykman et Roselyne Dégremont), Paris, GF-Flammarion, 1998.

DELEUZE, Gilles, Différence et répétition, Paris, PUF, 1968.

-, Empirisme et subjectivité, Paris, PUF, 1953.

—, L'île déserte et autres textes, Paris, Minuit, 2002.

—, Logique du sens, Paris, Minuit, 1969.

-, Nietzsche et la philosophie, Paris, PUF, 1962.

DELEUZE, Gilles, GUATTARI, Félix, Mille plateaux, Paris, Minuit, 1980.

-, Qu'est-ce que la philosophie?, Paris, Minuit, 1991.

DELEUZE, Gilles, PARNET, Claire, Dialogues, Paris, Flammarion, 1996.

HUME, David, Traité de la nature humaine. Livre I et appendice (traduction de Philippe Baranger et Philippe Saltel), Paris, GF-Flammarion, 1995.

HUSSERL, Edmund, Méditations cartésiennes (traduction de Marc De Launay), Paris, PUF, 1994.

KANT, Emmanuel, Critique de la raison pure (traduction de Alexandre J.L. Delamarre et François Marty, à partir de la traduction de Jules Barni), Paris, Gallimard, 1980.

LOCKE, John, Essai philosophique concernant l'entendement humain (traduction de Pierre Coste), Paris, Vrin, 1972.

PLATON, Sophiste (traduction de Nestor Cordero), Paris, GFFlammarion, 1993.

SAUVAGNARGUES, Anne, "Deleuze avec Bergson : le cours de 1960 sur L'évolution créatrice", dans WORMS, Frédéric (dir.), Annales bergsoniennes II, Paris, PUF, 2004, pp. 151-165.

1. Deleuze et Guattari, Qu'est-ce que la philosophie?, p. 8.

2. Deleuze, Nietzsche et la philosophie, p. 104.

3. Kant, Critique de la raison pure, pp. 579-597 (B707-732).

4. Deleuze et Guattari, Qu'est-ce que la philosophie?, p. 12. 
5. Deleuze, Différence et répétition, pp. 216-217. On retrouve déjà les germes de cette analyse dans le chapitre "Nouvelle image de la pensée" de Nietzsche et la philosophie, pp. 118-126.

6. Deleuze, Différence et répétition, pp. 44-45.

7. Aristote, Métaphysique, vol. I, p. 181 (?7, 1017a22-24).

8. Platon, Sophiste, pp. 120-121 (235d-236c).

9. Pour le détail de cette analyse : Deleuze, Différence et répétition, pp. 84-89; Deleuze et Guattari, Qu'est-ce que la philosophie?, pp. 33-34.

10. Deleuze, Différence et répétition, p. 79.

11. La position de Husserl (idéalisme intersubjectif) n'est pas tellement différente lorsque, pour fonder la "réalité» et l'objectivité du monde, il s'en tient à la présupposition que d'autres "Ego" auraient la même expérience que moi s'ils étaient à ma place (et vice versa). Husserl, Méditations cartésiennes, p. 172 (\$55). C'est alors l'expérience de la folie déréglant cette correspondance adéquate des «Ego" qui révèle la fragilité de ce fondement. Husserl peut bien dire que "l'anormalité doit d'abord elle-même se constituer comme telle, et elle ne le peut que sur le fond d'une normalité préalable» (Ibid., p. $175(\S 55)$ ), ce qui est ici interrogé, ce sont les conséquences de la volonté de fondement et non tel ou tel fondement en particulier. On comprend mieux du coup pourquoi Deleuze s'est intéressé à des artistes comme Antonin Artaud et Francis Bacon dont les oeuvres font voler en éclats ce "bon sens" et ce "sens commun".

12. Deleuze, Logique du sens, pp. 201-202.

13. Deleuze, Différence et répétition, p. 217.

14. C'est sur ce point que Deleuze prend ses distances d'avec Bergson, après avoir partagé avec lui ses griefs à l'encontre de la philosophie kantienne : alors que ce dernier étudie la création du point de vue de l'être (dualisme de la matière et de la durée et réduction ontologique de la première à la seconde), Deleuze réserve ce terme aux seuls produits de la penșée. Sauvagnargues, "Deleuze avec Bergson : le cours de 1960 sur L'évolution créatrice", pp. 163 164. Ainsi, pour Bergson, le domaine de la science est ontologiquement second par rapport à celui de la philosophie (il est le produit d'une abstraction), alors que pour Deleuze, l'un n'est pas l'antécédent de l'autre puisqu'il y une différence de nature irréductible entre leur activité et leur produit respectifs. Sur ces deux positions : Bergson, "L'intuition philosophique», La pensée et le mouvant, pp. 134-142; Deleuze et Guattari, "Fonctifs et concepts", Qu'est-ce que la philosophie?, pp. 111-127. II est vrai cependant que, dans Différence et répétition, Deleuze semble reprendre à son compte la critique bergsonienne de la valeur ontologique des "concepts" scientifiques, mais c'est seulement pour distinguer l'oeuvre spécifique de la philosophie d'une prétendue fondation objective de la science (par les catégories kantiennes par exemple). Voir à ce sujet : Bergson, "La perception du changement", La pensée et le mouvant, pp. 145-149; Deleuze, Différence et répétition, pp. 364-365.

15. Ibid., pp. 172-173.

16. Deleuze, Nietzsche et la philosophie, pp. 1-3.

17. Nous relevons ici une apparente ambiguitté dans l'utilisation de l'expression «Image de la pensée». Dans Différence et répétition (1968), Deleuze semble catégoriquement rejeter l'idée que la philosophie comme création de concepts puisse être, elle aussi, une "Image". Par contre, dans Qu'est-ce que la philosophie? (1991), il ne s'en prend pas à l' «Image» en tant que telle, mais oppose diverses "Images" entre 
elles. Dans les deux cas cependant, Deleuze s'attaque violemment aux différentes "Images" fondatrices qui ne peuvent, selon lui, qu'insuffler de la transcendance dans un exercice qui se voulait de prime abord immanent.

18. Deleuze, Différence et répétition, p. 79.

19. Ibid., p. 89.

20. Ibid., p. 286.

21. Ibid., p. 325.

22. Ibid., p. 44.

23. Ibid., p. 192.

24. Ibid., p. 306. Deleuze et Guattari, Qu'est-ce que la philosophie?, p. 87.

25. II y a quatre déformations possibles de la répétition formelle. Celles-ci correspondent aux quatre caractéristiques de la représentation précédemment analysées. Deleuze, Différence et répétition, pp. 346-349.

26. Ibid., p. 384.

27. Dans la philosophie kantienne par exemple, le schème assure le lien entre les deux instances hétérogènes que sont la sensibilité et l'entendement, mais Kant n'explique jamais comment ils en viennent tous deux à s'harmoniser dans le concept. Kant, Critique de la raison pure, pp. 192-193 (B180-181).

28. Deleuze, Différence et répétition, p. 165.

29. Pour le détail de cette «genèse du négatif» : Ibid., pp. 266-267.

30. Ibid., pp. 79-80.

31. Deleuze, «Entretien avec Gilbert [sic] Deleuze», Les Lettres françaises, $n^{\circ} 1223$ (28 février-5 mars 1968); repris dans L'île déserte et autres textes, p. 196.

32. Deleuze et Guattari, Qu'est-ce que la philosophie?, p. 49.

33. Voir par exemple : Deleuze, Différence et répétition, p. 4, p. 309; Deleuze, Logique du sens, p. 170.

34. Locke, Essai philosophique concernant l'entendement humain, pp. 1-2 (Avantpropos, §2 (Livre 1, 1er chapitre, §2 dans l'édition anglaise de référence)).

35. Ibid., p. 5 (Avant-propos, $§ 7$ (1.1.7 dans l'édition anglaise de référence)).

36. Ibid., p. 53 (1.3.20 (1.4.20 dans l'édition anglaise de référence)). Traduction légèrement modifiée.

37. Ibid., p. 91 (2.8.15). Traduction légèrement modifiée.

38. Ibid., p. 92 (2.8.17). Traduction légèrement modifiée.

39. Ibid., p. 9 (1.1.5 (1.2.5 dans l'édition anglaise de référence)).

40. Berkeley, Trois dialogues entre Hylas et Philonous, p. 207 (p. 246 dans l'édition anglaise de référence).

41. Berkeley, Principes de la connaissance humaine, p. 65 (1ère partie, §3).

42. Hume, Traité de la nature humaine. Livre l et appendice, p. 35 (Introduction, §8).

43. Ibid., p. 124 (Livre $1,2^{\theta}$ partie, $6^{\mathrm{e}}$ section, §8).

44. Ibid., p. 272 (1.4.2.4) et p. 297 (1.4.2.47).

45. Ibid., pp. $307-308$ (1.4.3.4) et p. 315 (1.4.4.6).

46. Locke, Essai philosophique concernant l'entendement humain, pp. 74-75 (2.1.25). Traduction légèrement modifiée. L'analogie avec le miroir (qui insinue que l'idée ressemble à l'objet) est probablement à mettre au compte de la croyance aux qualités premières que nous avons évoquée auparavant. 


\section{Charles Bolduc}

47. Ibid., p. 439 (4.3.1).

48. Ibid., p. 450 (4.3.10) et Hume, Traité de la nature humaine. Livre I et appendice, p. $211(1.3 .12 .20)$.

49. Locke, Essai philosophique concernant l'entendement humain, p. 70 (2.1.17).

50. Berkeley, Trois dialogues entre Hylas et Philonous, pp. 186-187 (p. 235 dans l'édition anglaise de référence).

51. Ibid., p. 147 (p. 215 dans l'édition anglaise de référence). Traduction légèrement modifiée.

52. Hume, Traité de la nature humaine. Livre I et appendice, p. 148 (1.3.5.6 (1.3.5.7 dans l'édition anglaise de référence qui insère dans le corps du texte les passages complémentaires publiés initialement en appendice)). Pour la définition du concept de croyance: Ibid., p. 161 (1.3.7.5).

53. Ibid., p. 377 (Appendice, §2 [1.3.7.7 dans l'édition anglaise de référence]).

54. Berkeley, Trois dialogues entre Hylas et Philonous, pp. 146-147 (pp. 214-215 dans l'édition anglaise de référence).

55. Ibid., p. 183 (p. 233 dans l'édition anglaise de référence).

56. Ibid., p. 221 (p. 253 dans l'édition anglaise de référence).

57. Hume, Traité de la nature humaine. Livre l et appendice, p. 344 (1.4.6.4).

58. Ibid., pp. 235-236 (1.3.14.10).

59. Même les démonstrations sont soumises à la probabilité : «Nous pouvons considérer notre jugement comme une sorte de cause, dont la vérité est l'effet naturel, mais un effet tel qu'il peut fréquemment être empêché par l'irruption d'autres causes ainsi que par l'inconstance des pouvoirs de notre esprit. La conséquence en est que toute connaissance dégénère en probabilité, et cette probabilité est plus ou moins grande suivant l'expérience que nous avons de la véracité ou du caractère trompeur de notre entendement, et aussi selon la simplicité ou la complexité de la question". Ibid., p. 261 (1.4.1.1).

60. Ibid., pp. 191-192 (1.3.10.8).

61. Par exemple, personne ne doute vraiment que César ait été assassiné aux ides de mars, même si personne aujourd'hui ne peut prétendre en avoir été le témoin direct. Ibid., pp. 144-145 (1.3.4.2).

62. Locke, Essai philosophique concernant l'entendement humain, pp. 553-554 (4.16.10).

63. Deleuze et Parnet, Dialogues, pp. 6970.

64. Hume, Traité de la nature humaine. Livre l et appendice, p. 231 (1.3.14.1). On peut raisonnablement penser que Deleuze était au fait de cette nuance : "L'effet de l'association dans les trois cas [relations de contiguilté, de ressemblance et de causalité] est le passage aisé de l'esprit d'une idée à une autre; l'essence de la relation, c'est la transition facile. L'esprit, devenu nature, a tendance». Deleuze, Empirisme et subjectivité, pp. 6-7.

65. Hume, Traité de la nature humaine. Livre I et appendice, p. 63 (1.1.7.3). Deleuze a bien souligné ce point : “L'association est une qualité qui unit les idées, non pas une qualité des idées elles-mêmes". Deleuze, 